\title{
Study of prostate cancer-derived extracellular vesicles in urine using IR spectroscopy
}

\author{
Xin-Le Yap ${ }^{1}$, Teng-Aik Ong ${ }^{2}$, Jasmine Lim², Bayden Wood ${ }^{3}$, Wai-Leng Lee ${ }^{1 *}$ \\ ${ }^{1}$ School of Science, Monash University Sunway Campus, Jalan Lagoon Selatan, Bandar Sunway, 47500 \\ Subang Jaya, Selangor, Malaysia. \\ ${ }^{2}$ Department of Surgery, Faculty of Medicine, University of Malaya, 50603 Kuala Lumpur, Malaysia. \\ ${ }^{3}$ Centre for Biospectroscopy and School of Chemistry, Monash University, Clayton, Victoria 3800 Aus- \\ tralia
}

\begin{abstract}
Prostate cancer ( $\mathrm{PCa}$ ) is the third most frequent cancer in men and prostate-specific antigen is currently the biomarker used despite its low specificity. Lately, extracellular vesicles (EVs) which are secreted by all types of cells have raised research interest for their association with cancer progression. Urinary EVs (UEVs) has emerged as a potential biomarker for $\mathrm{PCa}$ detection as it is non-invasive and urine samples are easily obtained from patients. Therefore, we hypothesize that PCa cells secrete EVs containing a unique set of biomolecules which can be exploited as a signature profile of the cancer. In this study, Attenuated Total Reflection-Fourier Transform Infrared (ATR-FTIR) spectroscopy was used for analysis of the UEVs aiming to obtain a signature spectrum for early detection of PCa. Urine samples from PCa and healthy subjects were subjected to ultracentrifugation for isolation of UEVs. Principal Component Analysis (PCA) indicated that FTIR spectra of the UEVs of PCa patients are distinct from those of healthy individuals at the following wavenumber values: amide I peak (1640 $\left.\mathrm{cm}^{-1}\right)$, RNA ribose peak $\left(1120 \mathrm{~cm}^{-1}\right), \mathrm{C}-\mathrm{C}, \mathrm{C}-\mathrm{N}$ stretch peak $\left(967 \mathrm{~cm}^{-1}\right)$ and $\mathrm{C} 4-\mathrm{C} 5 / \mathrm{C}=\mathrm{N}$, imidazole ring peak $\left(1610 \mathrm{~cm}^{-1}\right)$. The obtained IR spectra were also analyzed using Linear Discriminant Analysis (LDA) and the resulting diagnostic classifier for $\mathrm{PCa}$ achieved a sensitivity of $83.33 \%$ and a specificity of $60 \%$. In conclusion, ATR-FTIR analysis of UEVs in combine with PCA-LDA statistic model described in this study may offer a novel strategy for the development of a non-invasive urine test for early screening of prostate cancer.
\end{abstract}

Keywords: Extracellular vesicles; prostate cancer; FTIR; urine; biomarker

Received: $26^{\text {th }}$ March 2019

Accepted: $10^{\text {th }}$ May 2019

Published Online: $23^{\text {th }}$ July 2019

*Correspondence to: Lee Wai Leng, School of Science, Monash University Sunway Campus, Jalan Lagoon Selatan, Bandar Sunway, 47500 Subang Jaya, Selangor, Malaysia; lee.wai.leng@monash.edu

Citation: Yap XL, Ong TA, Lim J, et al. Study of prostate cancer-derived extracellular vesicles in urine using IR spectroscopy. Prog Drug Discov Biomed Sci 2019; 2(1): a0000026

\section{Introduction}

Prostate cancer is the third most frequent cancer in men worldwide ${ }^{[1]}$. Although it is relatively common compared to many other cancers, it has a 5-year survival rate as high as $98.6 \%{ }^{[1]}$. Prostate-specific antigen (PSA) is currently the most regular biomarker used in prostate cancer diagnosis despite its low specificity ${ }^{[2]}$. Increase in PSA levels was also observed in patients with benign prostatic hypertrophy. Many of such cases caused by the low specificity of PSA had led to the increasing numbers of unnecessary prostate biopsies ${ }^{[3]}$. Therefore, a biomarker with higher sensitivity and specificity that can be used for effective detection of the disease is urgently needed for better diagnosis of prostate cancer.

Extracellular vesicles (EVs) with a diameter range from $30-500 \mathrm{~nm}$, which are secreted by all types of cells in the body have raised research interest lately as they are found to be associated with malignancy. Increased secretion of EVs from cancer cells was observed and some of them were reported in building resistance to cancer treatment while some are contributing to the process of angiogenesis, an important attribute of a malignant tumor ${ }^{[4]}$. These vesicles contain lipids, proteins and nucleic acids such as mRNA and miRNA which are playing a significant role in intercellular communications ${ }^{[5,6]}$. Therefore, these biomolecules could potentially reflect the condition or status of their originating prostate cancer cells and provide important information about the progress of the disease. Several proteins ${ }^{[7,8]}$, mRNAs $^{[9]}$, microR$\mathrm{NAs}^{[10,11]}$ and lipids ${ }^{[12,13]}$ present in prostate cancer-derived EVs have the potential to become a biomarker for prostate cancer.

Compared to other body fluids such as blood and semen, urine sample is highly preferred for prostate cancer detection as the approach is non-invasive and the sample is easily obtained from the patients. Therefore, urinary extracellular vesicles have emerged as a potential biomarker for prostate cancer and numerous studies had been carried out to investigate its role in the progression 
of the disease $\mathrm{e}^{[7,9-11,14-18]}$. Various methods are used in the study of EVs. Immunoblotting ${ }^{[7,14,19-21]}$ is generally used as a method to identify the marker proteins expressed on the surface of the EVs for classification of the isolated vesicles. For imaging purpose, electron microscopy ${ }^{[7,9,11,21,22]}$ is used to verify the isolated EVs through their size and cup-shaped morphology. Mass spectrometry ${ }^{[7,21,22]}$ and $\mathrm{PCR}^{[9,11,19]}$ were also used to analyze the proteins and nucleic acids in EVs respectively. Despite all these established methods, infrared spectroscopy was rarely used in the study of EVs.

In our study, a work flow involves Attenuated Total Reflection Fourier Transform Infrared (ATR-FTIR) for the study on EVs isolated from urine samples was designed and optimized. The spectrum of a biological sample con- tains different absorption bands which represent proteins, lipids, nucleic acids, and others as a whole. As a result, IR spectroscopy could indicate the disease progress by detecting the changes of the molecular structure and composition of biopsy tissues ${ }^{[24]}$. ATR-FTIR can be used as a point-of-care diagnostic test in a clinic setting because it is very quick, portable and inexpensive. In addition, no chemical stains or reagents are required for the biochemical analysis of the sample ${ }^{[23]}$. The present work compared the absorbance spectra of EVs isolated from the urine of prostate cancer patients to those of normal individuals using ATR-FTIR. Figure 1 outlines the work flow for the acquisition of IR spectra of urinary EVs and derivation of diagnostic classifier for PCa detection via spectra analysis.

\section{Method details}

Urine sample collection

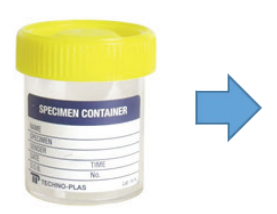

Low-speed centrifugation

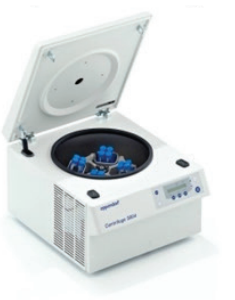

Ultracentrifugation

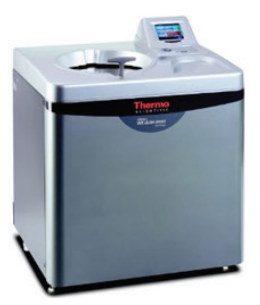

EV pellet
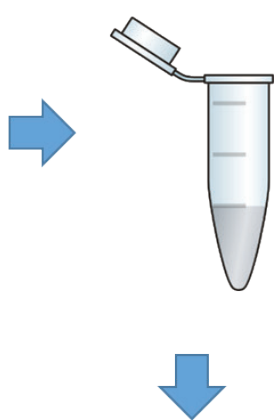

Spectral Acquisition

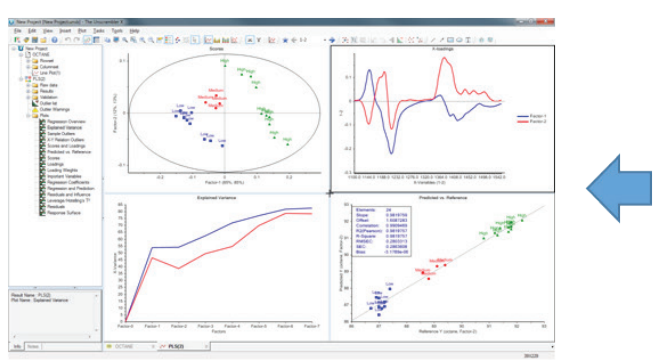

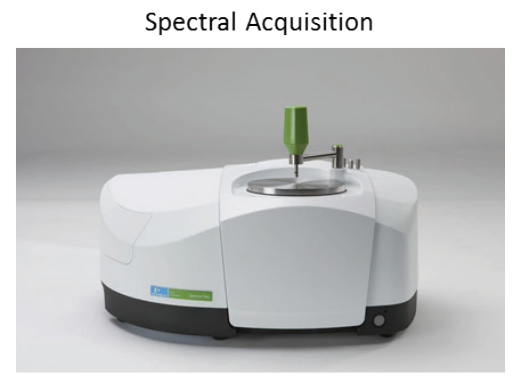

Figure 1. Schematic flow chart of the protocol of isolation of urinary extracellular vesicles and ATR-FTIR spectra data analysis

\section{$\underline{\text { Isolation of urinary extracellular vesicles }}$}

- Materials

- Urine preserved in sodium azide

- $\quad$ Phosphate-buffered saline (PBS)

- Dithiothreitol (DTT) (1M) in ultrapure water

- 50-ml polypropylene centrifuge tubes

- Ultracentrifuge and fixed-angle or swingingbucket rotor

- Polyallomer tubes or polycarbonate bottles

\section{Procedure}

Preserved urine samples should be stored at $-80{ }^{\circ} \mathrm{C}$ and to be moved to $4{ }^{\circ} \mathrm{C}$ storage 1 day prior to urinary extracellular vesicles isolation.

1. Vortex the urine samples for 30 seconds each. Transfer the fluid into 50-ml tubes.

2. Centrifuge the urine samples at $400 \mathrm{x} g$ and $15,500 \mathrm{x}$ $\mathrm{g}$ for 20 minutes respectively.
3. Carefully transfer the supernatant to ultracentrifuge tubes and spin at 200,000 x g using a fixed-angle rotor for 90 minutes at $18^{\circ} \mathrm{C}$.

4. Discard the supernatant and resuspend the pellet using 0.5 $\mathrm{ml}$ of $1 \mathrm{M}$ DTT and $1.5 \mathrm{ml}$ of PBS.

5. Incubate the suspensions at $37^{\circ} \mathrm{C}$ for 10 minutes.

6. Top up the suspensions with PBS and centrifuge the samples at 200,000 x g using a swinging- bucket rotor for 70 minutes at $25{ }^{\circ} \mathrm{C}$.

7. Pour away the supernatant and resuspend the pellet using $100 \mu 1$ of PBS to the pellet.

8. Store at $-80^{\circ} \mathrm{C}$ until further analysis.

Analysis of urinary extracellular vesicles using Attenuated Total Reflection-Fourier Transform Infrared (ATR-FTIR) Spectroscopy

\section{Materials}

- $\quad 0.45 \mu \mathrm{m}$ Ultrafree-MC Centrifugal Filter (Merck) 
- $\quad$ Phosphate-buffered saline (PBS)

- Ultrapure water

- Blow dryer

- $\quad$ ATR-FTIR Spectroscopy

- The Unscrambler X 10.5.1 software

\section{Procedure}

To study the difference in IR spectra between the 2 groups, their IR spectra are collected for further statistical analysis.

1. Filter the urinary extracellular samples using a 0.45 $\mu \mathrm{m}$ Ultrafree-MC Centrifugal Filter (Merck).

2. Set the settings of the FTIR machine to 32 scans, 4 $\mathrm{cm}^{-1}$ resolution and wavelength from $4000 \mathrm{~cm}^{-1}$ to 400 $\mathrm{cm}^{-1}$.

3. Acquire a background spectrum.

4. Acquire the spectrum of ultrapure water and PBS.

5. Clean the diamond ATR crystal with isopropanol.

6. Load $2 \mu \mathrm{l}$ of the filtrate on the diamond ATR crystal.

7. Blow dry the solution on the diamond using a blow dryer.

8. Run triplicates for each sample.

9. Save the spectrum files (.sp format) and proceed to analyze using the Unscrambler software.

10. Import all the extracellular vesicles spectra into the software (.sp format).

11. Correct baseline by performing Baseline Correction.

12. Perform Unit Vector Normalization on the spectra.

13. Apply Derivative Savitsky Golay transformation at the following parameters:

- Derivative Order: 1

- Polynomial Order: 2

- $\quad$ Smoothing Points: 21

- Left Point: 10

- Right Points: 10

14. Apply Standard Normal Variate transformation.

15. Apply Principal Component Analysis (PCA) to the wavelength ranged between $1794-813 \mathrm{~cm}^{-1}$ at the following parameters:

- Number of PCs: 7

- Validation type: Cross validation

- Apply Linear Discriminant Analysis (LDA) to the same batch of samples.

\section{Method Validation}

\section{$\underline{\text { Isolation of urinary extracellular vesicles }}$}

To evaluate the presence and characteristics of extracellular vesicles in the isolate, various downstream applications were carried out to determine their size, morphology and surface protein markers. The results of these applications demonstrated that the isolation protocol generated a group of heterozygous urinary extracellular vesicles which are round and membranous in shape, diameter ranged within 30 - $500 \mathrm{~nm}$ and contain detectable EV surface protein markers such as CD9 and CD10.

Analysis of urinary extracellular vesicles using Attenuated Total Reflection-Fourier Transform Infrared (ATR-FTIR) Spectroscopy

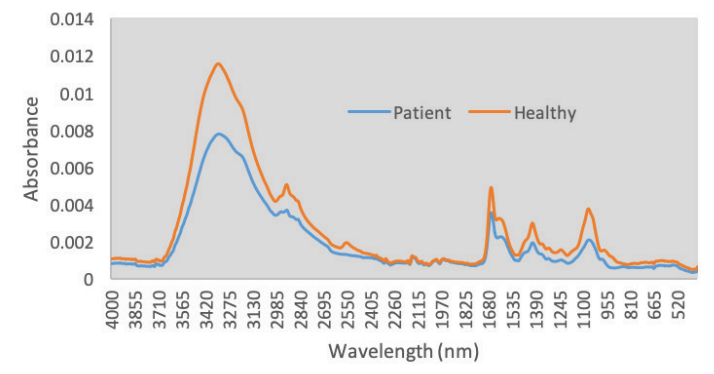

Figure 2. The average spectra of Healthy $(\mathrm{n}=5)$ and Patient $(\mathrm{n}=6)$ samples

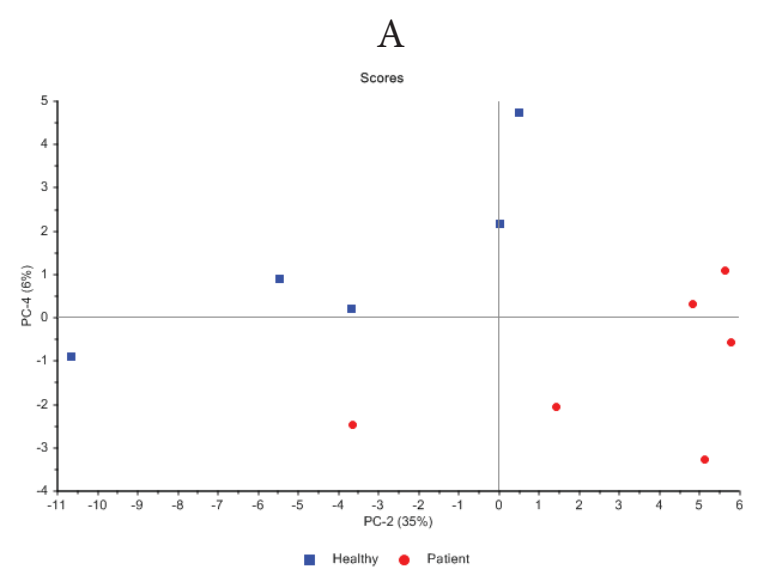

B

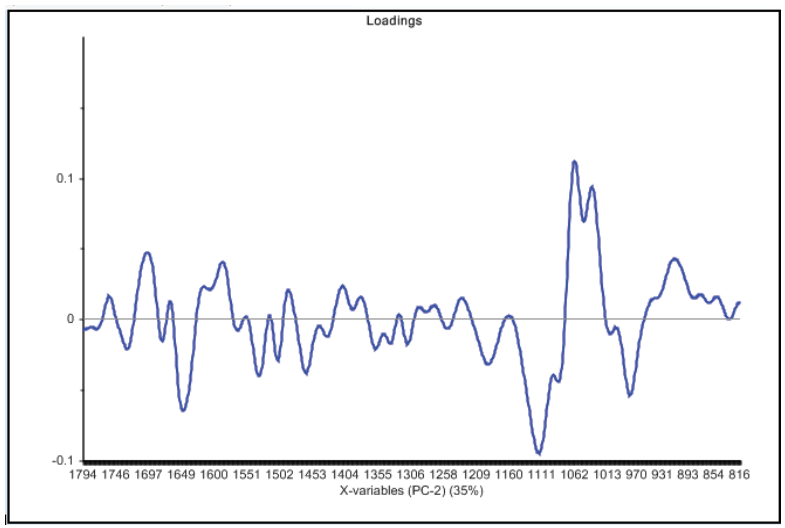

C

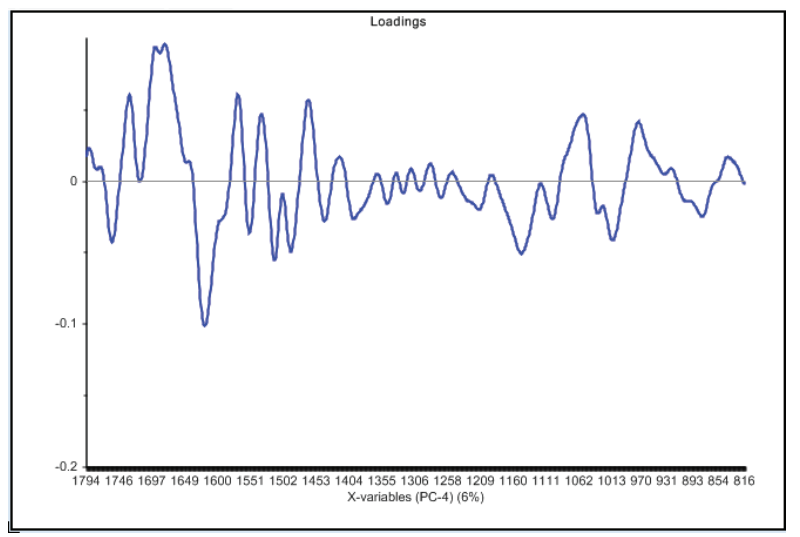

Figure 3. Principal component analysis of the subsets of EVs pre-processed by Unscrambler X. (A) Score plot of the second and forth PCs, with the corresponding percentage of explained variance in parentheses. (B) Loadings of the second PC with $35 \%$ of explained variance. (C) Loadings of the forth PC with $6 \%$ of explained variance. 
Based on Figure 2, the average spectra of patient EV samples has a lower absorbance compared to healthy EV samples at the fingerprint region. After analysing the spectra using Principal Component Analysis (PCA), the 2 groups showed spectral peak differences at amide I peak $(1640 \mathrm{~cm}-$ 1), RNA ribose peak (1120 cm-1), C-C, C-N stretch peak $(967 \mathrm{~cm}-1)$ and $\mathrm{C} 4-\mathrm{C} 5 / \mathrm{C}=\mathrm{N}$, imidazole ring peak $(\sim 1610$ $\mathrm{cm}-1$ ) (Figure 3 ). These peaks could then possibly be used as biomarkers for identifying prostate-derived UEVs of prostate cancer patients. The spectra obtained were used to derive a diagnostic classifier for PCa using Linear Discriminant Analysis (LDA) and the resulting diagnostic classifier for PCa achieved a sensitivity of $83.33 \%$ and a specificity of $60 \%$. The difference in wavenumber values between 2 groups and the analysis using LDA-derived classifier indicates the ATR-FTIR technique has potential utility as a point-of-care test for PCa in urine. In conclusion, ATR-FTIR analysis of UEVs in combine with PCA-LDA statistic model described in this study may offer a novel strategy for the development of a non-invasive urine test for early screening of prostate cancer.

\section{Ethics Statement}

This experiment was approved by the Medical Research Ethics Committee of University of Malaya Medical Centre (MREC ID NO: 2017728-5442) and Monash University Human Research Ethics Committee (Project ID: 13232).

\section{Conflict of Interest}

There is no conflict of interest.

\section{Acknowledgements}

This research was financially supported by Monash Malaysia Large Strategic Research Grant Scheme and Fundamental Research Grant Scheme grants (FRGS/1/2014/ SKK01/MUSM/03/2) from the Ministry of Higher Education Malaysia.

\section{Reference}

1. Howlader NNA, Krapcho M, Miller D, et al, (eds), SEER Cancer Statistics Review.

2. Chou R, et al. Screening for prostate cancer: a review of the evidence for the U.S. Preventive Services Task Force. Ann Intern Med 2011 155(11): 762-71.

3. Stephan C, et al. Prostate-Specific Antigen (PSA) Screening and New Biomarkers for Prostate Cancer (PCa). EJIFCC 2014; 25(1): 55-78.

4. Zhang J, et al. Exosome and Exosomal MicroRNA: Trafficking, Sorting, and Function. Genomics, Proteomics \& Bioinformatics 2015; 13(1): 17-24.

5. Kalluri R. The biology and function of exosomes in cancer. J Clin Invest 2016; 126(4): 1208-1215.

6. Liu CM, et al. Exosomes from the tumor microenvironment as reciprocal regulators that enhance prostate cancer progression. International Journal of Urology 2016; 23(9): 734-744

7. Overbye A, et al. Identification of prostate cancer biomarkers in urinary exosomes. Oncotarget 2015; 6(30): 30357-30376.

8. Fujita K, et al. Proteomic analysis of urinary extracellular vesicles from high Gleason score prostate cancer. Sci Rep 2017; 7: 42961.

9. Nilsson J, et al. Prostate cancer-derived urine exosomes: a novel approach to biomarkers for prostate cancer. Br J Cancer 2009; 100(10): 1603-1607.

10. Carter DRF, et al. Comparative Study of Extracellular Vesicles from the Urine of Healthy Individuals and Prostate Cancer Patients. Plos One 2016; 11(6)

11. Foj L, et al. Exosomal and Non-Exosomal Urinary miRNAs in Prostate Cancer Detection and Prognosis. Prostate 2017; 77(6): 573-583.

12. Brzozowski JS, et al. Lipidomic profiling of extracellular vesicles derived from prostate and prostate cancer cell lines. Lipids in health and disease 2018; 17(1): 211-212.

13. Skotland T, et al. Molecular lipid species in urinary exosomes as potential prostate cancer biomarkers. Eur Journal Cancer 2017; 70: 122-132.

14. Mitchell PJ, et al. Can urinary exosomes act as treatment response markers in prostate cancer? J Transl Med 2009; 7: 4

15. Mizutani K, et al. Isolation of prostate cancer-related exosomes. Anticancer Res 2014; 34(7): 3419-3423.

16. Motamedinia P, et al. Urine Exosomes for Non-Invasive Assessment of Gene Expression and Mutations of Prostate Cancer. PLoS One 2016; 11(5): e0154507.

17. Tavoosidana $\mathrm{G}$, et al. Multiple recognition assay reveals prostasomes as promising plasma biomarkers for prostate cancer. Proc Natl Acad Sci U S A 2011; 108(21): 8809-8814.

18. Zijlstra C, Stoorvogel W. Prostasomes as a source of diagnostic biomarkers for prostate cancer. J Clin Invest 2016; 126(4): 1144-1151.

19. Alvarez ML, et al. Comparison of protein, microRNA, and mRNA yields using different methods of urinary exosome isolation for the discovery of kidney disease biomarkers. Kidney Int 2012; 82(9): 1024-1032.

20. Zhou H, et al. Collection, storage, preservation, and normalization of human urinary exosomes for biomarker discovery. Kidney Int 2006; 69(8): 1471-1476

21. Pisitkun T, Shen RF, Knepper MA. Identification and proteomic profiling of exosomes in human urine. Proc Natl Acad Sci U S A 2004; 101(36): 13368-13373.

22. Wang Z, et al. Proteomic analysis of urine exosomes by multidimensional protein identification technology (MudPIT). Proteomics 2012; 12(2): 329-338.

23. Kneipp J, et al. Molecular changes of preclinical scrapie can be detected by infrared spectroscopy. J Neurosci 2002; 22(8): 2989-2997.

24. Heraud $\mathrm{P}$, et al. Early detection of the chemical changes occurring during the induction and prevention of autoimmune-mediated demyelination detected by FT-IR imaging. NeuroImage 2010; 49(2): 1180-1189. 